Research Paper

\title{
LATS1 exerts tumor suppressor functions via targeting Glil in colorectal cancer
}

\author{
Zhengchao Shen ${ }^{1 \#, ~ Y i n g y i n g ~ P a n ~}{ }^{2 \#}$, Peng Chen ${ }^{1}$, Bin Jiang ${ }^{1}$, Xiaosan Fang ${ }^{1}$ and Yaqi Jiang ${ }^{1}$ \\ 1. Department of Hepatobiliary Surgery, The First Affiliated Hospital of Wannan Medical College, Wuhu, Anhui, 241000, P.R. China. \\ 2. Department of Urinary Surgery, The Second Affiliated Hospital of Wannan Medical College, Wuhu, Anhui, 241000, P.R. China. \\ \#These authors contributed equally to this work. \\ $\square$ Corresponding author: Dr. Yaqi Jiang, Department of Hepatobiliary Surgery, The First Affiliated Hospital of Wannan Medical College, Wuhu, Anhui, 241000, \\ P.R. China. E-mail: jiangyaqi2021@126.com.
}

() The author(s). This is an open access article distributed under the terms of the Creative Commons Attribution License (https://creativecommons.org/licenses/by/4.0/). See http:/ /ivyspring.com/terms for full terms and conditions.

Received: 2021.04.30; Accepted: 2021.10.04; Published: 2021.10.28

\begin{abstract}
Background: The Hippo pathway's primary kinase component, large tumor suppressor 1 (LATSI), has been hypothesized as a tumor suppressor in a variety of cancers. LATSI's biological effects on colorectal cancer (CRC) are yet to be determined.

Methods: The analysis of LATS1 mRNA expression in CRC was conducted using public databases from the Gene Expressing Profiling Interactive Analysis database (GEPIA). Investigation for the expression of LATS1 protein in 102 CRC tumor tissues and 57 normal tissues was performed using immunohistochemistry (IHC) analysis. In vitro genetic manipulation was used to explore the potential role and mechanism of LATS1 in the regulation of proliferation and migration of CRC cells.

Results: LATS1 was found to be considerably downregulated in CRC tissues, with much lower levels in individuals with bigger tumors of size $(\geq 5 \mathrm{~cm})$, deeper invasion (T3-4), positive lymph node metastasis (LNM), and advanced tumor-node-metastasis (TNM) stage (III-IV). As exhibited by clinical data analysis, LATS1 loss was significantly associated with TNM and LNM staging in CRC patients. Furthermore, our in vitro investigations revealed that LATS1 depletion increased CRC cell proliferation and migration in HCT 116 cells, whereas overexpressing LATS1 had the opposite effect in SW620 cells. LATS1 suppressed the expression of glioma-associated oncogene-1 (Gli1), and LATSI's tumor-suppressive actions in CRC are dependent on Glil. Moreover, LATSI could modulate Yes-associated protein 1 (YAPI) expression and mTOR activation in CRC cells.

Conclusion: Our findings identify the LATS1 as a unique Glil regulator in CRC cell migration and proliferation, and suggest that LATSI may serve as a potential therapeutic target for CRC.
\end{abstract}

Key words: LATS1, proliferation, migration, Gli1, colorectal cancer

\section{Introduction}

Colorectal cancer (CRC) is one of the most common cancers worldwide, and it is still the third biggest cause of cancer-related mortality and morbidity [1-3]. Despite the fact that individuals with CRC who are discovered early on have an overall survival rate of around $80 \%$, over one-third of patients will die from their disease, primarily due to distant metastases [3]. Emerging data confirm the involvement of several genes and cellular signaling pathways in the development and metastasis of CRC [4]. Therefore, discovering new targets and clarifying the underlying biochemical pathways could lead to more effective CRC therapy techniques.

The Hippo pathway regulates various biological processes which include cell apoptosis, proliferation, and differentiation, to control tissue and organ size [5, 6]. In mammalian systems, the core components of this pathway comprise the mammalian STE20-like protein kinase 1/2 (MST1/2), the large tumor suppressor 1/2 (LATS1/2), and their downstream transcription co-activators, Yes-associated protein 1 (YAP1) and TAZ [7, 8]. MST1/2 kinase activates 
LATS1/ 2 by the process of phosphorylation, which leads to the phosphorylation of YAP1/TAZ and supplies a docking site for the 14-3-3 protein, causing cytoplasmic retention and ubiquitin-mediated proteasomal degradation of YAP1 and TAZ [5]. Recently, accumulating evidence has suggested that perturbation of the Hippo pathway contributes to cancer development [5-9]. LATS1, as the major kinase component of the Hippo pathway, exerts a significant role to control tumor cell proliferation, growth, and migration [10-13]. It was suggested that it could be used as a tumor suppressor in a variety of cancers including breast carcinoma [14, 15], ovarian cancer [16], non-small cell lung cancer [17], hepatocellular carcinoma [18], cervical cancer [19] and gastric cancer [20]. In CRC, LATS1 protein expression was downregulated and its decreased expression was associated with promoter hypermethylation [21]. The exact functional significance and fundamental mechanisms of LATS1 in CRC, however, are unknown and need to be investigated.

The Hedgehog signaling system is known to be involved in the genesis and progression of malignancies, including CRC [22, 23]. Smoothened (SMO), known as the canonical Hedgehog pathway, can activate Gli1, a major transcription factor in the Hedgehog signaling pathway [24]. It can also be activated by protein kinase B (AKT), extracellular signal-regulated kinase (ERK), and mammalian target of rapamycin (mTOR) which is known as the non-canonical Hedgehog pathway [24, 25].

Herein, LATS1 expression in CRC tissues was investigated, as well as the relationship between LATS1 protein expression and clinicopathological variables. LATS1's biological roles in CRC were also investigated using the loss of function and gain of function studies. Moreover, our findings revealed new mechanical insights into the critical functions of LATS1 in reducing Gli1 via modulating YAP1 expression and mTOR activation, potentially implying new CRC treatment options.

\section{Materials and methods}

\section{Clinical data and tissue samples}

The relevant clinical data were gathered from the Gene Expressing Profiling Interactive Analysis database (GEPIA) which can be found at http:// gepia.cancer-pku.cn/. Formalin-fixed and fresh tissue samples were acquired from Wannan Medical College's First Affiliated Hospital and verified by pathological diagnosis. Prior to surgery, none of the patients had chemo or radiotherapies. This study was approved by the Institute Research Medical Ethics Committee of Wannan Medical College's First
Affiliated Hospital. In compliance with the Declaration of Helsinki, all subjects supplied written informed consent. Table 1 summarizes the characteristics of the patients.

Table 1. Relationship between LATSI protein expression (detected by $\mathrm{IHC}$ ) and clinic-pathological factors in $102 \mathrm{CRC}$ patients

\begin{tabular}{|c|c|c|c|c|c|}
\hline \multirow[t]{2}{*}{ Clinic parameters } & \multirow[t]{2}{*}{ Case No. } & \multicolumn{2}{|c|}{ LATS1 expression } & \multirow[t]{2}{*}{$\chi^{2}$} & \multirow[t]{2}{*}{$P$ value } \\
\hline & & None or low & High & & \\
\hline Total & 102 & 37 & 65 & & \\
\hline \multicolumn{6}{|l|}{ Age (years) } \\
\hline$<65$ & 37 & 15 & 22 & 0.457 & 0.499 \\
\hline$\geq 65$ & 65 & 22 & 43 & & \\
\hline \multicolumn{6}{|l|}{ Gender } \\
\hline Male & 68 & 22 & 46 & 1.357 & 0.244 \\
\hline Female & 34 & 15 & 19 & & \\
\hline \multicolumn{6}{|l|}{ Tumor size } \\
\hline$<5 \mathrm{~cm}$ & 53 & 15 & 38 & 3.034 & 0.082 \\
\hline$\geq 5 \mathrm{~cm}$ & 49 & 22 & 27 & & \\
\hline \multicolumn{6}{|l|}{ Tumor location } \\
\hline Left-sided colon & 32 & 12 & 20 & 1.955 & 0.376 \\
\hline Right-sided colon & 33 & 9 & 24 & & \\
\hline Rectum & 37 & 16 & 21 & & \\
\hline \multicolumn{6}{|c|}{ Degree of differentiation } \\
\hline Well & 89 & 32 & 57 & & 1.000 \\
\hline Poor & 13 & 5 & 8 & & \\
\hline \multicolumn{6}{|l|}{ Depth of invasion } \\
\hline T1-2 & 17 & 3 & 14 & 3.062 & 0.080 \\
\hline T3-4 & 85 & 34 & 51 & & \\
\hline \multicolumn{6}{|c|}{ Lymph node metastasis } \\
\hline Yes & 42 & 23 & 19 & 10.557 & $0.001^{* *}$ \\
\hline No & 60 & 14 & 46 & & \\
\hline \multicolumn{6}{|l|}{ TNM stage } \\
\hline I-II & 59 & 14 & 45 & 9.529 & $0.002^{* *}$ \\
\hline III-IV & 43 & 23 & 20 & & \\
\hline
\end{tabular}

\section{Immunohistochemistry (IHC)}

IHC staining of paraffin-embedded human tissues was carried out as reported previously [25, 26]. In brief, Samples were dewaxed, rehydrated and endogenous peroxidase activity was blocked with 3\% hydrogen dioxide. The sections were incubated with the primary antibodies recognizing human LATS1 (dilution 1:100; \#17049-1-AP, Proteintech) overnight at $4{ }^{\circ} \mathrm{C}$, and with the secondary antibody for 1 hour at room temperature. Finally, the sections were visualized using a tissue staining kit (Zhongshanjinqiao Biotechnology, Beijing, China). LATS1 protein expression were scored according to the percentage of positive cells and the staining intensity. Percentage score was assigned as $1(<25 \%)$, $2(25-50 \%), 3(51-75 \%), 4(76-100 \%)$. The staining intensity was graded into 0 (no staining), 1 (week), 2 (moderate), and 3 (strong). A final score was calculated by the multiple of staining percentage and intensity. The LATS1 protein levels were considered as high when the final scores were 5 or above, and as none or low expression when the final scores were $<5$. 


\section{Cell culture and transfection}

Chinese Academy of Sciences Committee Type Culture Collection cell bank (Shanghai, China) was provided the human CRC cell lines (HCT8, HCT116, LoVo, RKO, SW480, SW620, CaCo2, and DLD-1). We maintained the cells in 10\% fetal bovine serum (FBS) supplemented RPMI 1640 medium (Gibco, USA) containing $1 \%$ penicillin/streptomycin (P/S; Gibco, USA) at $37{ }^{\circ} \mathrm{C}$ with a $5 \% \mathrm{CO}_{2}$ atmosphere. The Lentivirus vectors carrying LATS1 overexpression or short hairpin RNA (shRNA) targeting LATS1 and their control vectors were invented by Genechem Company (Shanghai, China). We performed the transfection following the company's instructions, which were documented in our earlier study [25, 26]. Lipofectamine RNAiMax (Invitrogen) was used to transfect small interfering RNA (siRNA) at $20 \mathrm{nM}$ of final concentration, as directed by the manufacturer. The shRNA sequence targeting human LATS1 is 5'-ATCCTCGACGAGAGCAGA-3'. The siRNA sequence targeting human Gli1 is 5'-CUCCACAGGC AUACAGGAU-3'.

\section{Western blot analysis and Protein extraction}

RIPA lysis buffer (Beyotime, China) was used for the extraction of total proteins, and the determination of protein content was carried out with a BCA protein assay kit (Pierce, USA). The protein separation was performed on an $8-10 \%$ polyacrylamide gel by sodium dodecyl sulphate polyacrylamide gel electrophoresis (SDS-PAGE) and then transferred to polyvinylidene difluoride (PVDF) membranes. Chemiluminescence was used to visualize the proteins after they had been incubated with secondary and primary antibodies. The bands were quantified using the Imaging J analysis software. The detailed protocol was described previously [25, 26]. Following antibodies used in this study: anti-LATS1 (dilution 1:1000; \#17049-1-AP, Proteintech), anti-Gli1 (dilution 1:1000; \#ab217326, Abcam), anti-YAP1 (dilution 1:1000; \#14074, Cell Signaling Technology (CST)), anti-p-mTOR (dilution 1:1000; \#9205, CST), anti-mTOR (dilution 1:1000; \#2983, CST), anti-GAPDH (dilution 1:5000; \#AG019, Beyotime).

\section{RNA extraction and quantitative real-time PCR (qRT-PCR)}

Total RNA was prepared as per the manufacturer's instructions using TRIzol reagent (Thermo Fisher Scientific). cDNA was synthesized and amplified by PCR using specified primers. qPT-PCR was performed on ABI 7500 Fast Real-Time PCR System (Applied Biosystems, UK) with SYBR Green RT-PCR kits (ABI, USA). As an endogenous control, the human GAPDH were used. The study used the following primers: human-LATS1-Forward, GTT AAG GGG AGA GCC AGG TCCTT, humanLATS1-Reverse, TCA AGG AAG TCC CCA GGA CTGT; human-GAPDH-Forward, AAT CCC ATC ACC ATC TTC, human-GAPDH-Reverse, AGG CTG TTG TCA TAC TTC [26].

\section{Assay for migration and cell viability}

Colony formation and Transwell migration assays were used to identify cell proliferation and migration ability respectively by using the protocol described previously [25, 27].

\section{Statistical analysis}

Means \pm standard errors of the mean (SEM) are used to express the data. The experimental results were statistically evaluated using two-tailed Student's $t$-test or one-way analysis of variance (ANOVA). The chi-square statistical test or Fisher's exact test was used to examine the IHC results. For all tests, a $P$-value of $<0.05$ was considered statistically significant.

\section{Results}

\section{Expression of LATS1 is decreased in human CRC tissues}

We first investigated the mRNA expression of LATS1 via the GEPIA database, and the results showed decreased mRNA level of LATS1 in CRC tumor tissues (T) compared to the normal controls $(\mathrm{N}$, Figure 1A). To further determine the role of LATS1 in colorectal tumorigenesis, an IHC assay was conducted in human para-cancer normal and CRC tissues. IHC staining revealed that the positive LATS1 expression rates were $82.5 \%(47 / 57)$ in normal colorectal tissues and $63.7 \%(65 / 102)$ in CRC tissue samples (Figure 1B, Table 1). When compared to normal tissue samples, the IHC score of LATS1 staining in CRC tissue samples was lower $(P<0.001$, Figure 1C). Furthermore, LATS1 protein expression levels in CRC tumor tissues with larger tumor sizes $(\geq 5 \mathrm{~cm}$ ), deeper invasion (T3-4), positive lymph node metastasis (LNM), or advanced tumor-nodemetastasis (TNM) stage (III-IV) were lower significantly as compared to control groups (Figure 1D-G). However, there was no link found between LATS1 protein expression and tumor site or differentiation level (Figure $1 \mathrm{H}$ and I).

The relationship between LATS1 protein expression and clinicopathological features in CRC tumor tissues was then investigated further. Chisquare test analysis showed the negative correlation of LATS1 protein expression with LNM $(P=0.001)$ and TNM staging $(P=0.002)$, but no correlation was observed with other clinicopathological factors such 
as tumor size, gender, age, tumor location, depth of invasion and degree of differentiation $(P>0.05$, Table $1)$.

\section{LATSI expression in human CRC cell lines}

By using western blot and qRT-PCR analyses, we detected the LATS1 expression in eight human CRC cell lines (HCT8, HCT116, LoVo, RKO, SW480, SW620, $\mathrm{CaCo} 2$, and DLD-1) (Figure 2A and 2B respectively). Compared with the low expression of LATS1 at both mRNA and protein levels in SW620 and $\mathrm{CaCo} 2$ cell lines, other cell lines (especially HCT116 and SW480) showed a relatively high concentration of LATS1 (Figure 2A and B). Hence, we used a lentivirus-based shRNA approach to knock down LATS1 expression in HCT116 and SW480 cells, which had significant amounts of endogenous LATS1 protein and mRNA. LATS1 expression was lesser in cells transfected with LATS1-targeting shRNA (KD) than in cells transfected with control-shRNA (NC, $P$ $<0.001$ ) (Figure 2C and 2D representing the result of Western blot and qRT-PCR respectively). Similarly, we used both analyses to examine the overexpression effectiveness of human LATS1 encoded with plasmid or empty vector in $\mathrm{SW} 620$ and $\mathrm{CaCo} 2$ cells that produced relatively low endogenous LATS1 (Western Blot, Figure 2E) and (qRT-PCR, Figure 2F). The results showed that cells transfected with plasmids containing human LATS1 (OE) expressed more LATS1 protein and mRNA than cells transfected with empty vector (VEC, $P<0.001$ ).
A

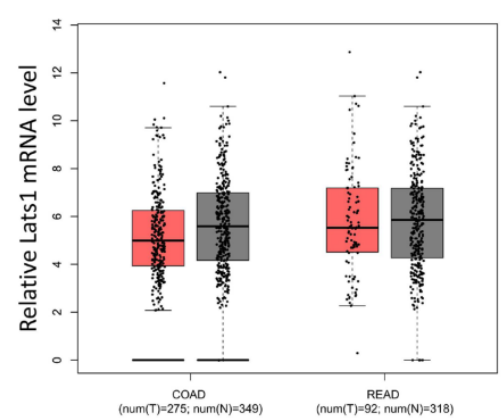

D

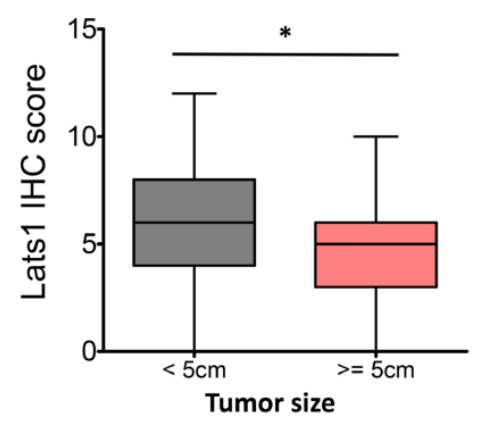

G

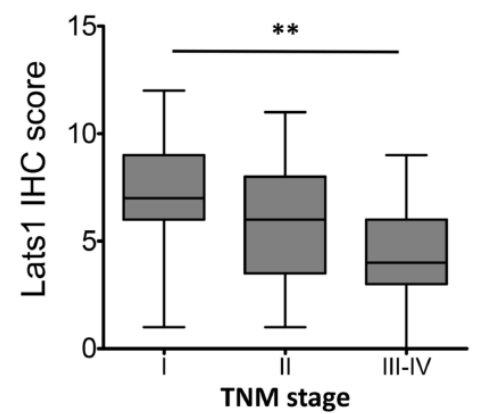

B

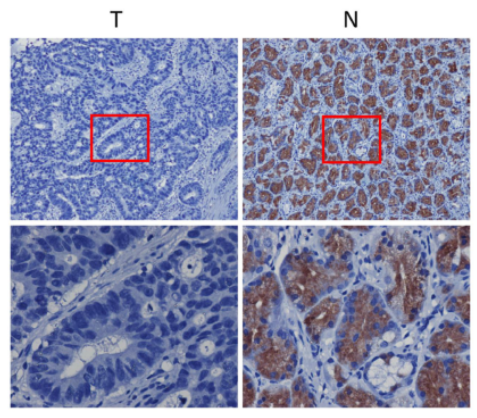

E

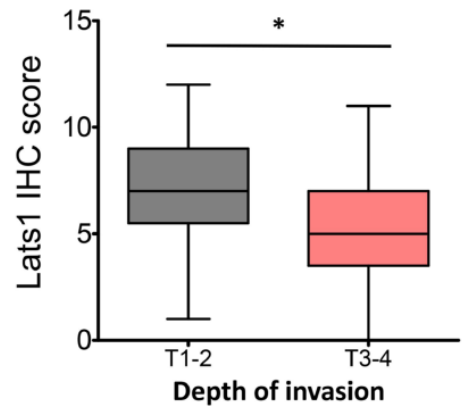

H

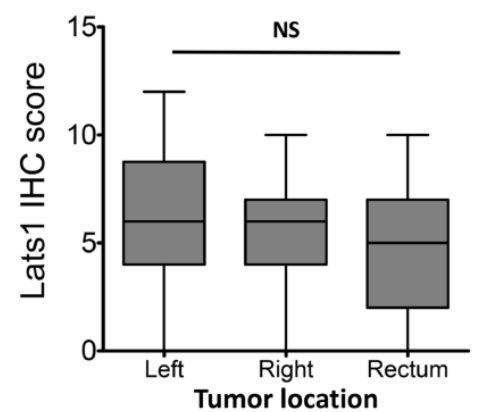

C

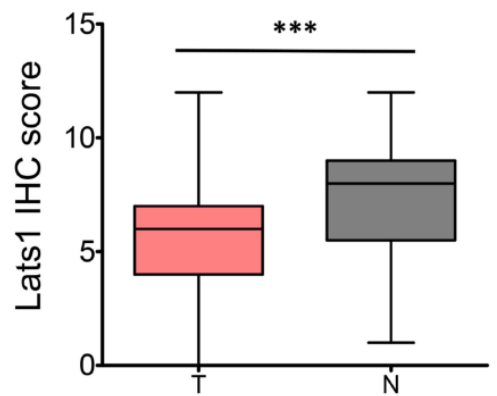

F

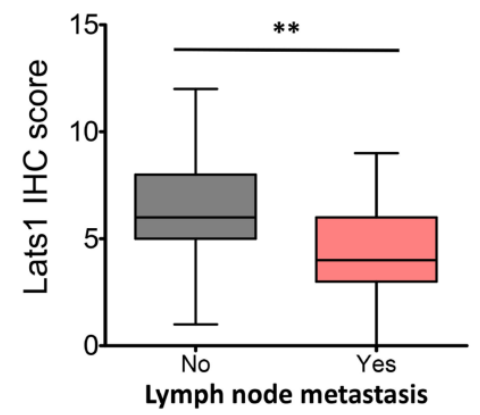

I

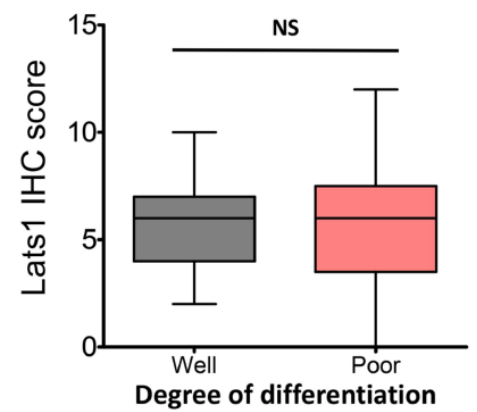

Figure 1. LATS1 expression in human CRC tissues. (A) Data from the GEPIA database showed that LATS1 mRNA was downregulated in CRC tumor tissues (T) compared to normal controls (N), COAD colon adenocarcinoma, READ rectal adenocarcinoma. (B) IHC staining for LATS1 in CRC tissues and peritumoural normal tissues. (C) The IHC score of LATS1 in CRC tissue samples (T) and normal tissues (N). (D-I) LATS1 expression in CRC tumor tissues of different tumor size (D), depth of invasion (E), lymph node metastasis $(\mathbf{F})$, TNM stage $(\mathbf{G})$, tumor location $(\mathbf{H})$ and degree of differentiation $(\mathrm{I})$. NS, nonsignificant, $* P<0.05$, $* * P<0.01, * * * P<0.001$. 
A

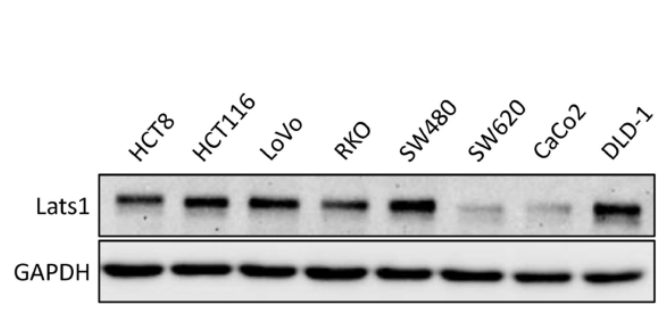

B

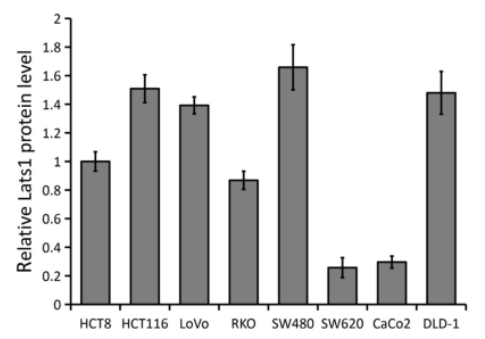

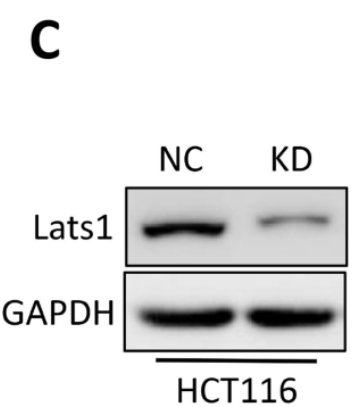

HCT116

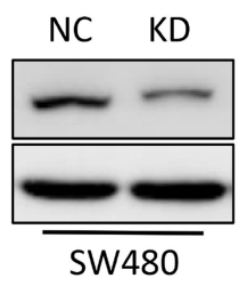

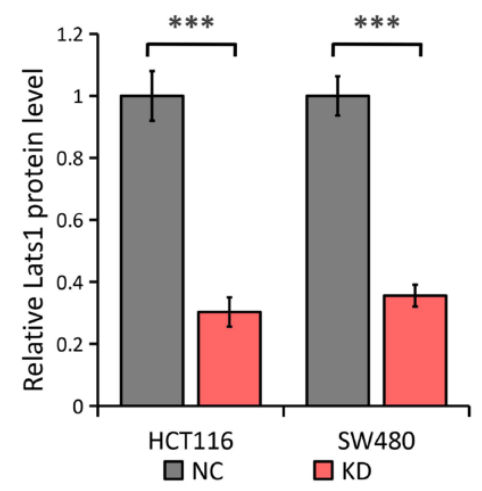
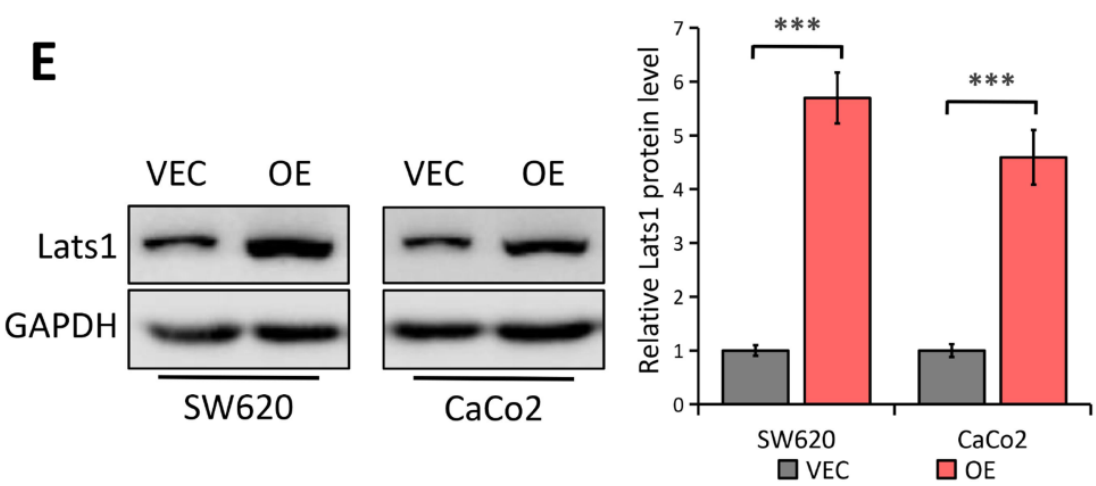
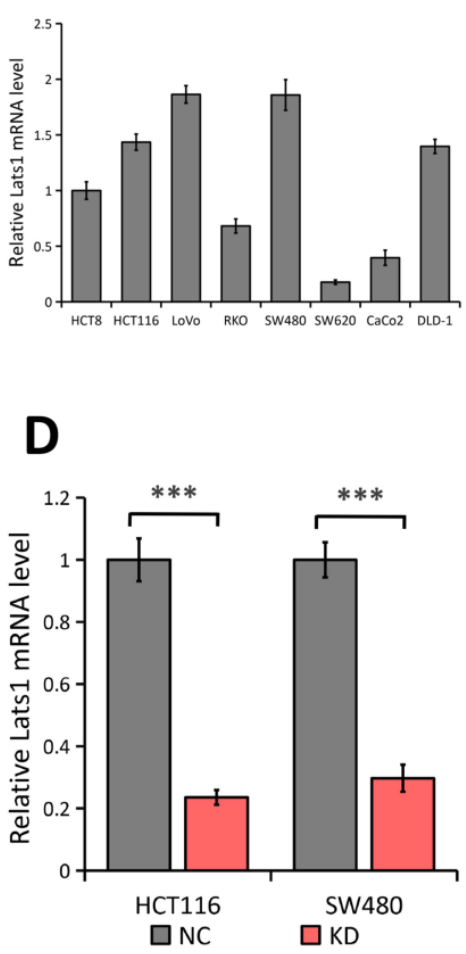

$\mathbf{F}$

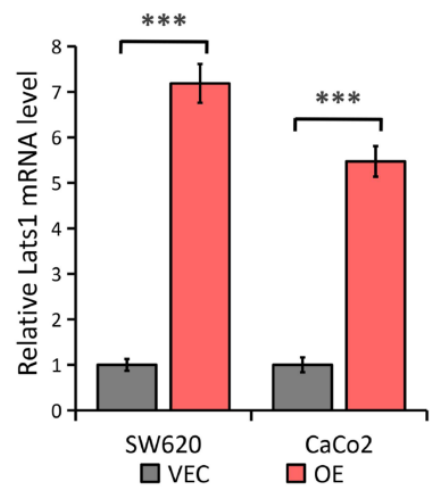

Figure 2. LATS1 expression in human CRC cell lines. (A-B) Western blot (A) and qRT-PCR (B) analysis of LATS1 expression in CRC cell lines (HCT8, HCT116, LoVo, RKO, SW480, SW620, CaCo2 and DLD-1). (C-D) Western blot (C) and qRT-PCR (D) analysis of LATS1 expression in HCT116 and SW480 cells stably transfected with control-shRNA (NC) or shRNA against LATS1 (KD). (E-F) Western blot (E) and qRT-PCR (F) analysis of LATS1 expression in SW620 and CaCo2 cells stably transfected with empty vector (VEC) or plasmids encoding human LATS1 (OE). $* * * P<0.001$.

\section{LATSI inhibits the proliferation and migration of CRC cells}

Since the LATS1 expression was reduced in CRC tissues, we next investigated the potential biological functions of LATS1 in CRC cells. A colony formation experiment was used to see if LATS1 influences the proliferation of CRC cells. The results revealed that the colony number of HCT116 cells dramatically increased following depletion of LATS1 $(P<0.01$, Figure 3A), while the foci forming potential of SW620 cells was significantly reduced under elevated LATS1 level $(P<0.01$, Figure 3B). We then used a transwell test to see how LATS1 affected cell migration. The analysis indicates that reducing LATS1 amplified the capacity of HCT116 cells to migrate $(P<0.01$, Figure $3 C)$. In contrast to the control group, HCT116 cells with stable LATS1 overexpression demonstrated a significant reduction in migratory cells $(P<0.01$, Figure 3D).

\section{The tumor-suppressive function of LATS 1 is dependent on Glil}

We predicted that Gli1 might be involved in LATS1-mediated inhibition of CRC cell to proliferate and migrate, given that abnormal Gli1 expression in the Hedgehog pathway promotes CRC development $[28,29]$. LATS1 knockdown enhanced the expression 
of Gli1 in SW480 and HCT116 cells $(P<0.01$; Figure $4 \mathrm{~A})$. Conversely, the levels of Gli1 protein were significantly decreased in LATS1-overexpressing cells $(P<0.01$, Figure 4B) indicating the suppression of Gli1under the influence of LATS1 in CRC cells.

We employed Gli1-specific siRNA (siGli1) to knock down the expression of Gli1 in HCT116 cells with or without LATS1 depletion to see if Gli1 is involved in LATS1-mediated reduction of cell proliferation and migration in CRC. According to the Western blot analysis, the Gli1 protein level was decreased in cells transfected with siGli1 as compared to the control group (Figure 4C). In colony formation and transwell migration assays, LATS1 knockdown considerably boosted the proliferation and migratory ability of HCT116 cells $(P<0.01$, Figure $4 \mathrm{D}$ and E), but the enhanced proliferation and migration was greatly impaired when Gli1 was silenced $(P>0.05)$.

\section{LATS1 regulates YAPI expression and $M T O R$ activation in CRC cells}

In esophageal squamous cell carcinoma, YAP1 has been shown to upregulate Gli1 [30], which is in accordance with our previous study in gastric cancer [30], representing that YAP1 enhances cell migration and proliferation via regulating Gli1 expression through the AKT/mTOR signaling pathway. It was also reported that LATS1 suppresses mTOR activity [15] and activated mTOR can enhance Gli1 expression in an SMO-independent manner [24]. Considering LATS1 as a critical regulator of the YAP1 signaling [5], we speculated that YAP1/mTOR signaling might be implicated in LATS1 inhibition of Gli1. The Western
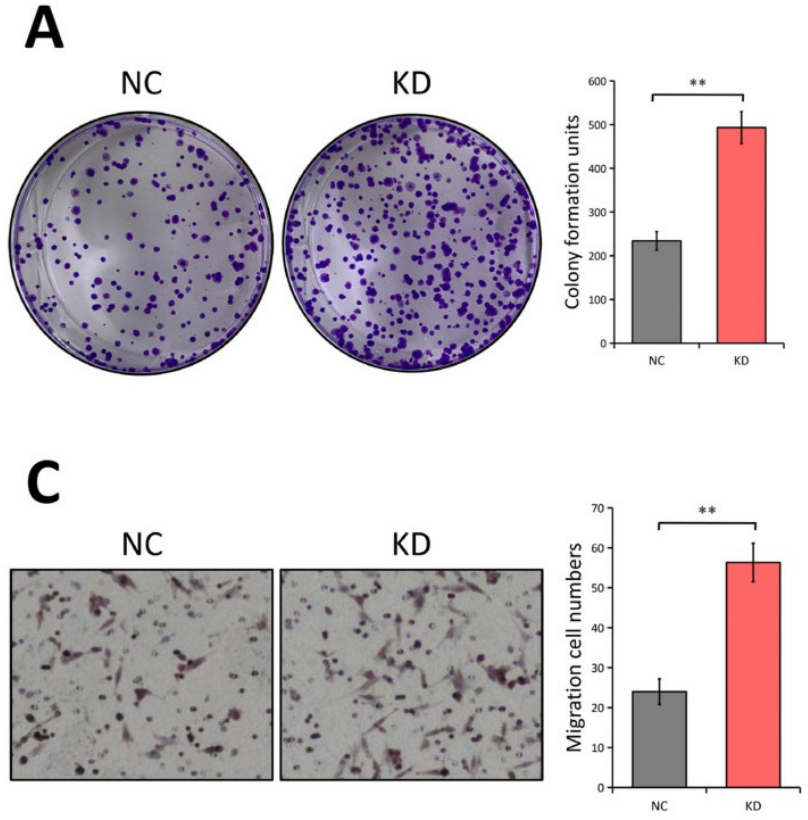

blot analysis showed that LATS1 depletion notably promoted YAP1 expression and mTOR phosphorylation in both SW480 and HCT116 cells $(P<$ 0.05 , Figure $5 \mathrm{~A})$, with no effect on the total mTOR level. On the contrary, LATS1 elevation inhibited YAP1 expression and mTOR activity in SW620 and $\mathrm{CaCo} 2$ cells $(P<0.05$, Figure 5B). Our findings suggest that LATS1 regulates the YAP1/mTOR signaling pathway in CRC cells, which could explain the reason for Gli1 suppression.

\section{Discussion}

The Hippo pathway's primary kinase component, LATS1, has been hypothesized as a tumor suppressor in a variety of cancers [14-20]. LATS1 protein expression was downregulated in CRC, and this was linked to promoter hypermethylation [21], although the specific functional aspects and underlying mechanisms of LATS1 in CRC remain unknown.

In this work, we evidenced the downregulation of LATS1 expression in human CRC which was reduced further in patients with positive LNM, and advanced TNM stage (III-IV). Furthermore, clinical data analysis revealed that loss of LATS1 protein expression has a significant association with TNM and LNM staging in CRC patients. Moreover, in vitro gain of function and loss of function tests revealed that silencing LATS1 dramatically increased the CRC cell's potential to proliferate and migrate, whereas LATS1 overexpression did the opposite, signifying the tumor-suppressive effects of LATS1.

B
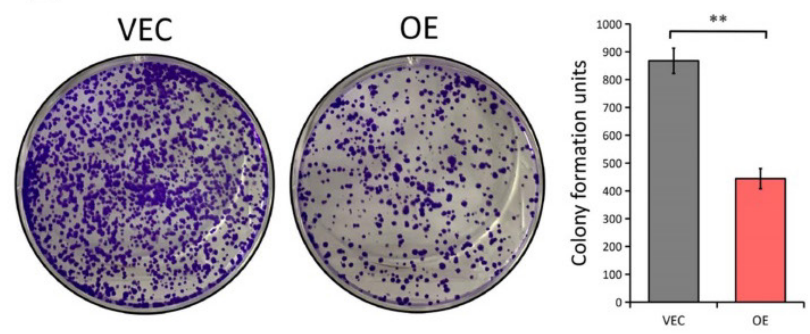

D
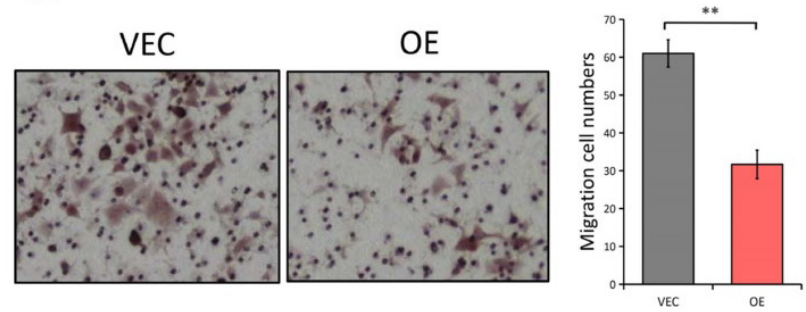

Figure 3. LATS1 plays tumor-suppresive roles in CRC. (A) Colony formation assay in HCT116 cells stably transfected with control-shRNA (NC) or shRNA against LATS1 (KD). (B) Colony formation assay in SW620 cells stably transfected with empty vector (VEC) or plasmids encoding human LATS1 (OE). (C) Transwell assay in HCT116 cells (NC vs. KD). (D) Transwell assay in SW620 cells (VEC vs. OE). $* * P<0.01$. 
A

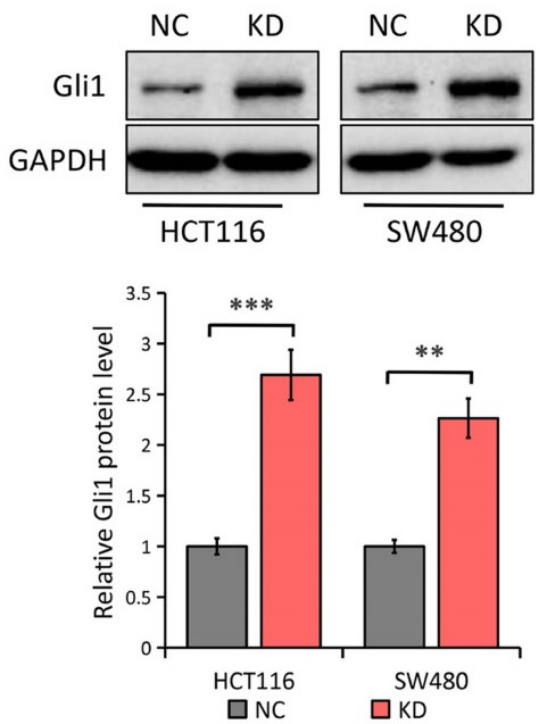

D

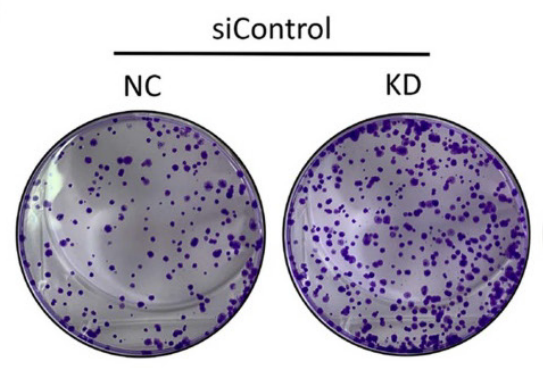

$\mathbf{E}$

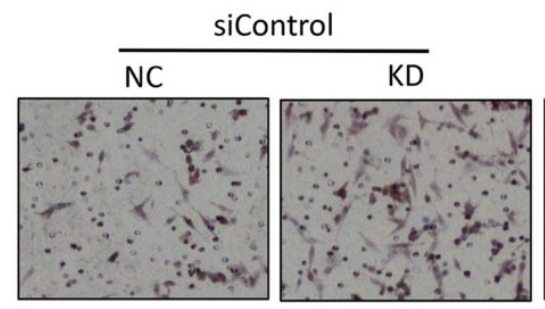

B
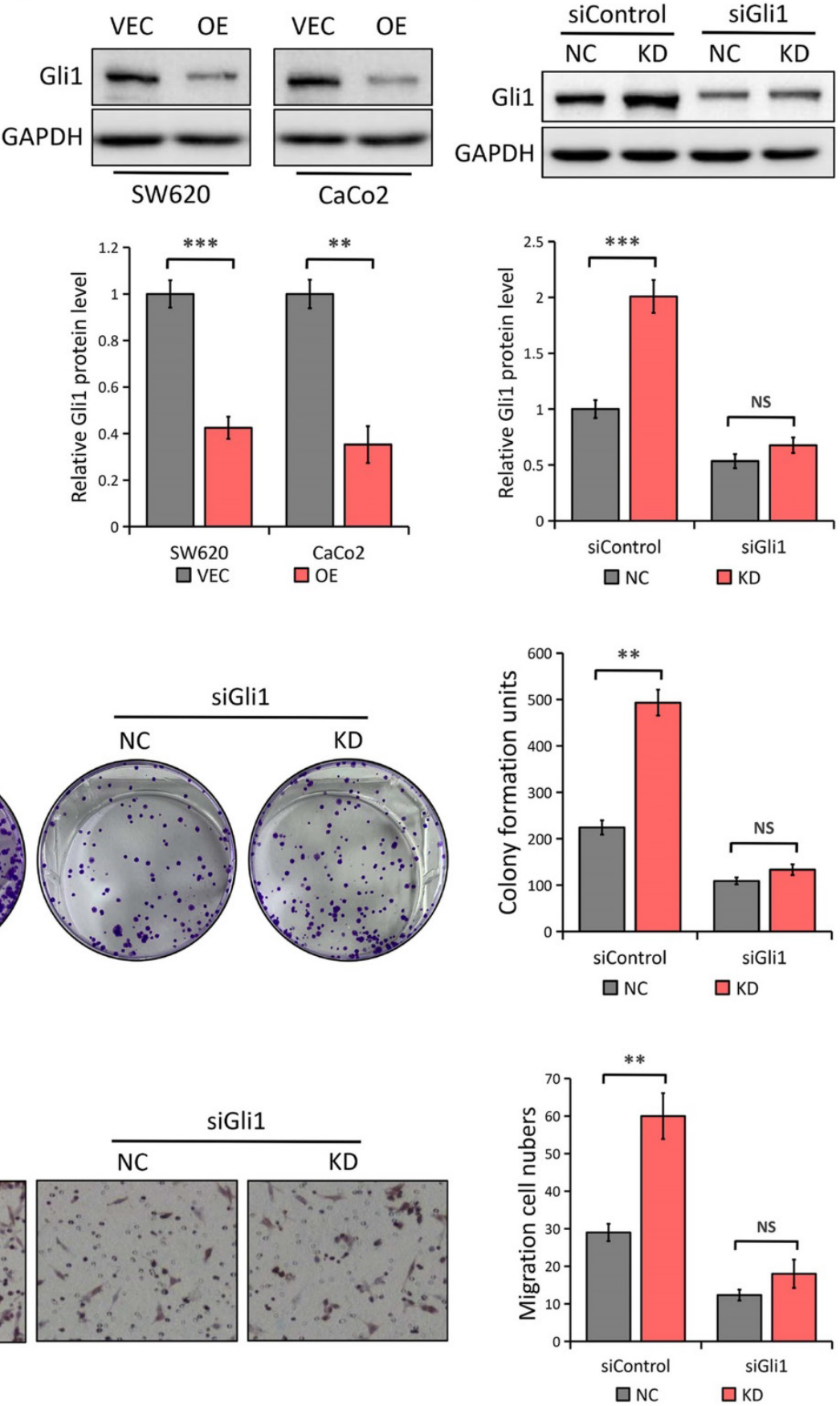

Figure 4. LATS1 inhibits CRC cell proliferation and migration in a Glil-dependent manner. (A) Western blot analysis of Gli1 expression in HCT116 and SW480 cells stably transfected with control-shRNA (NC) or shRNA against LATS1 (KD). (B) Western blot analysis of Glil expression in SW620 and CaCo2 cells stably transfected with empty vector (VEC) or plasmids encoding human LATS1 (OE). (C) Western blot analysis of Gli1 expression in LATS1-depleted HCT116 cells with or without Gli1 siRNA treatment. (D) Colony formation assay in LATS1-depleted HCT116 cells with or without Gli1 siRNA treatment. (E) Transwell assay in LATS1-depleted HCT116 cells with or without Glil siRNA treatment. NS, nonsignificant, $* * p<0.01$, $* * * P<0.001$.

Gli1, as a key transcription factor of the Hedgehog signaling pathway, exerts a critical role in CRC development $[28,29]$. Our findings imply that LATS1 regulates Gli1 expression in CRC, as authenticated by the fact that LATS1 knockdown enhanced the expression of Gli1 while LATS1 elevation did the opposite. Gli1 was silenced in HCT116 cells with or without LATS1 depletion to see if it was involved in LATS1 regulation of CRC cell proliferation and migration and it was observed that LATS1 knockdown promoted the migration and proliferation of HCT116 cells, but the enhanced proliferation and migration ability was greatly impaired when Gli1 was depleted, indicating the dependence of anti-carcinogenic roles of LATS1 on Gli1. 

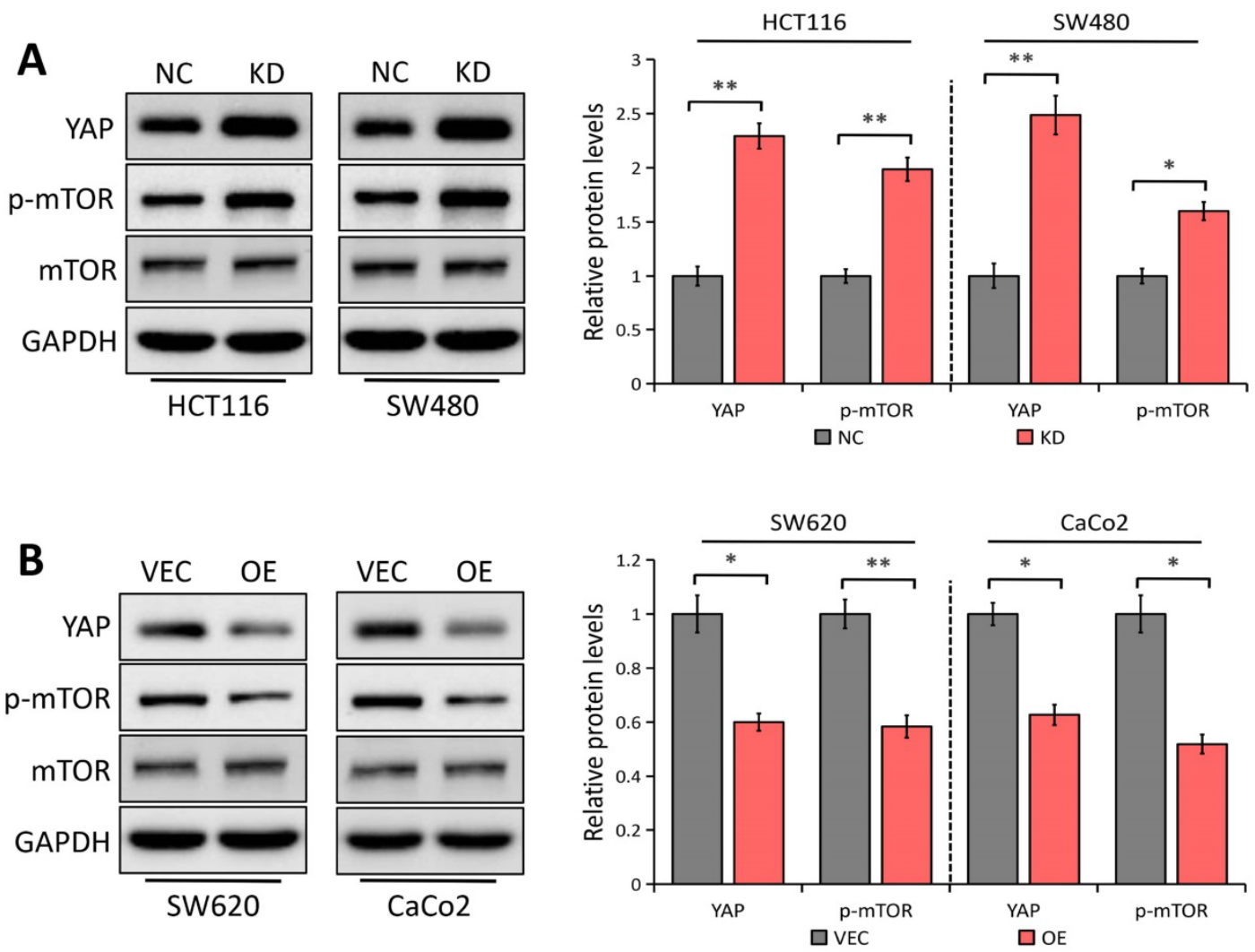

Figure 5. LATSI regulates YAPI expression and mTOR signaling pathway. (A) Western blot analysis of the indicated proteins in HCT116 and SW480 cells stably transfected with control-shRNA (NC) or shRNA against LATSI (KD). (B) Western blot analysis of the indicated proteins in SW620 and CaCo2 cells stably transfected with empty vector (VEC) or plasmids encoding human LATSI (OE). $* P<0.05, * * P<0.01$.

Given the facts that LATS1 is a critical regulator of YAP1 [5] and YAP1 can upregulate Gli1 expression in esophageal squamous cell carcinoma [30] and gastric cancer [31], we speculated that YAP1 might be implicated in LATS1-mediated Gli1 suppression. Here, we confirmed that LATS1 depletion promoted YAP1 expression while LATS1 elevation inhibited YAP1 expression in CRC cells. Furthermore, it was recently discovered that LATS1 reduces mTOR activity to directly coordinate the mTORC1 and Hippo pathways in growth control [15] and activated mTOR can enhance Gli1 expression [24], we asked whether the mTOR signaling is involved in LATS1regulated Gli1 inhibition. The results showed that LATS1 depletion notably promoted mTOR phosphorylation in both HCT116 and SW480 cells, without affecting the expression level. On the contrary, LATS1 elevation inhibited mTOR activity in SW620 and CaCo2 cells. Moreover, another possible mechanism is that LATS1 elevation decreased YAP1 protein level, leading to mTOR inactivation, which subsequently suppressed Gli1 expression. Collectively, findings suggest that LATS1 works as a regulator of the YAP1/mTOR signaling pathway in CRC cells, and is capable to cause Gli1 suppression.

Although more in-depth studies are needed to clarify the mechanisms regarding the Gli1 regulation by LATS1 in CRC, our results strongly confirmed that the LATS1 is a tumor suppressor in CRC. Furthermore, we discovered a putative role for LATS1 to regulate the CRC cell proliferation and migration by direct or indirect inhibiting Gli1. This could open up a new avenue for CRC treatment by targeting the LATS1/Gli1 signaling axis.

\section{Acknowledgements}

The authors would like to thank all the reviewers who participated in the review and MJEditor (www. mjeditor.com) for its linguistic assistance during the preparation of this manuscript.

\section{Authors' contributions}

Zhengchao Shen and Yingying Pan planned, designed and performed the experiments and wrote the manuscript. Peng Chen, Bin Jiang and Xiaosan Fang contributed to data collection and analysis. Yaqi Jiang supervised, planned, and designed the research and wrote the manuscript. All authors have read and agreed to the current version of the manuscript.

\section{Competing Interests}

The authors have declared that no competing interest exists. 


\section{References}

1. Tfaily MA, Naamani D, Kassir A, Sleiman S, Ouattara M, Moacdieh MP, Jaffa MA. Awareness of colorectal cancer and attitudes towards its screening guidelines in Lebanon. Ann Global Health. 2019; 85: 1-11.

2. N. Keum, E. Giovannucci. Global burden of colorectal cancer: emerging trends, risk factors and prevention strategies. Nat Rev Gastroenterol Hepatol. 2019; 16: 713-732.

3. Siegel RL, Miller KD, Fedewa SA, Ahnen DJ, Meester RGS, Barzi A, Jemal A. Colorectal cancer statistics, 2017. CA Cancer J Clin. 2017; 67: 177-193.

4. Grady, W. M. \& Markowitz, S. D. The molecular pathogenesis of colorectal cancer and its potential application to colorectal cancer screening. Dig. Dis. Sci. 2015; 60: 762-772.

5. Moroishi T, Hansen CG, Guan KL. The emerging roles of YAP and TAZ in cancer. Nat Rev Cancer. 2015; 15(2): 73-79.

6. Yu FX, Zhao B, Guan KL. Hippo pathway in organ size control, tissue homeostasis, and cancer. Cell. 2015; 163: 811-828.

7. Mo JS, Meng Z, Kim YC, Park HW, Hansen CG, Kim S, Lim DS, Guan KL. Cellular energy stress induces AMPK-mediated regulation of YAP and the Hippo pathway. Nat Cell Biol. 2015; 17(4): 500-510.

8. Wang W, Xiao ZD, Li X, Aziz KE, Gan B, Johnson RL, Chen J. AMPK modulates Hippo pathway activity to regulate energy homeostasis. Nat Cell Biol. 2015; 17(4): 490-499.

9. Harvey KF, Zhang X, Thomas DM. The Hippo pathway and human cancer. Nat. Rev. Cancer. 2013; 13: 246-257.

10. Yu T, Bachman J, Lai ZC. Evidence for a tumor suppressor role for the large tumor suppressor genes LATS1 and LATS2 in human cancer. Genetics. 2013; 195: 1193-1196.

11. Yabuta N, Mukai S, Okamoto A, Okuzaki D, Suzuki H, Torigata K, Yoshida K, Okada N, Miura D, Ito A, Ikawa M, Okabe M, Nojima H. N-terminal truncation of Lats1 causes abnormal cell growth control and chromosomal instability. J Cell Sci. 2013; 126: 508-520.

12. Huntoon CJ, Nye MD, Geng L, Peterson KL, Flatten KS, Haluska P, Kaufmann SH, Karnitz LM. Heat shock protein 90 inhibition depletes LATS1 and LATS2, two regulators of the mammalian hippo tumor suppressor pathway. Cancer Res. 2010; 70: 8642-8650.

13. Furth N, Bossel Ben-Moshe N, Pozniak Y, Porat Z, Geiger T, Domany E, Aylon $Y$, Oren M. Down-regulation of LATS kinases alters p53 to promote cell migration. Genes Dev. 2015; 29: 2325-2330.

14. Britschgi A, Duss S, Kim S, Couto JP, Brinkhaus H, Koren S, De Silva D, Mertz KD, Kaup D, Varga Z, Voshol H, issieres A, Leroy C, Roloff T, Stadler MB, Scheel CH, Miraglia LJ, Orth AP, Bonamy GM, Reddy VA, Bentires-Alj M. The Hippo kinases LATS1 and 2 control human breast cell fate via crosstalk with ERa. Nature. 2017; 541(7638): 541-545.

15. Gan W, Dai X, Dai X, Xie J, Yin S, Zhu J, Wang C, Liu Y, Guo J, Wang M, Liu J, Hu J, Quinton RJ, Ganem NJ, Liu P, Asara JM, Pandolfi PP, Yang Y, He Z, Gao G, Wei W. LATS suppresses mTORC1 activity to directly coordinate Hippo and mTORC1 pathways in growth control. Nat Cell Biol. 2020; 22(2): 246-256.

16. Yagi H, Onoyama I, Asanoma K, Hori E, Yasunaga M, Kodama K, Kijima M, Ohgami T, Kaneki E, Okugawa K, Yahata H, Kato K. Ga13-mediated LATS1 down-regulation contributes to epithelial-mesenchymal transition in ovarian cancer. FASEB J. 2019; 33(12): 13683-13694.

17. Luo SY, Kwok HH, Yang PC, Ip MS, Minna JD, Lam DC. Expression of large tumour suppressor (LATS) kinases modulates chemotherapy response in advanced non-small cell lung cancer. Transl Lung Cancer Res. 2020; 9(2): 294-305.

18. Tang F, Gao R, Jeevan-Raj B, Wyss CB, Kalathur RKR, Piscuoglio S, Ng CKY, Hindupur SK, Nuciforo S, Dazert E, Bock T, Song S, Buechel D, Morini MF, Hergovich A, Matthias P, Lim DS, Terracciano LM, Heim MH, Hall MN, Christofori G. LATS1 but not LATS2 represses autophagy by a kinaseindependent scaffold function. Nat Commun. 2019; 10(1): 5755.

19. Deng J, Zhang W, Liu S, An H, Tan L, Ma L. LATS1 suppresses proliferation and invasion of cervical cancer. Mol Med Rep. 2017; 15(4): 1654-1660.

20. Zhang J, Wang G, Chu SJ, Zhu JS, Zhang R, Lu WW, Xia LQ, Lu YM, Da W, Sun $\mathrm{Q}$. Loss of large tumor suppressor 1 promotes growth and metastasis of gastric cancer cells through upregulation of the YAP signaling. Oncotarget. 2016; 7(13): 16180-93

21. Wierzbicki PM, Adrych K, Kartanowicz D, Stanislawowski M, Kowalczyk A Godlewski J, Skwierz-Bogdanska I, Celinski K, Gach T, Kulig J, Korybalski B, Kmiec Z. Underexpression of LATS1 TSG in colorectal cancer is associated with promoter hypermethylation. World J Gastroenterol. 2013; 19(27): 4363-73.

22. Taipale J, Beachy PA. The Hedgehog and Wnt signalling pathways in cancer. Nature. 2011; 411: 349-354.

23. Park SH, Jeong S, Kim BR, Jeong YA, Kim JL, Na YJ, Jo MJ, Yun HK, Kim DY, Kim BG, Lee DH, Oh SC. Activating CCT2 triggers Gli-1 activation during hypoxic condition in colorectal cancer. Oncogene. 2020; 39(1): 136-150.

24. Wang Y, Ding Q, Yen CJ, Xia W, Izzo JG, Lang JY, Li CW, Hsu JL, Miller SA, Wang X, Lee DF, Hsu JM,Huo L, Labaff AM, Liu D, Huang TH, Lai CC, Tsai FJ, Chang WC, Chen CH, Wu TT, Buttar NS, Wang KK, Wu Y, Wang H, Ajani J, Hung MC. The crosstalk of mTOR/S6K1 and Hedgehog pathways. Cancer Cell. 2012; 21(3): 374-387.

25. Song L, Guo X, Zhao F, Wang W, Zhao Z, Jin L, Wu C, Yao J, Ma Z. TTC36 inactivation induced malignant properties via Wnt- $\beta$-catenin pathway in gastric carcinoma. J Cancer. 2021; 12(9): 2598-2609.
26. Xu M, Wang J, Li H, Zhang Z, Cheng Z. AIM2 inhibits colorectal cancer cell proliferation and migration through suppression of Gli1. Aging (Albany NY). 2021; 13: 1017-1031.

27. Cheng Z, Shao X, Xu M, Wang J, Kuai X, Zhang L, Wu J, Zhou C, Mao J. Rab1A promotes proliferation and migration abilities via regulation of the HER2/ AKT-independent mTOR/S6K1 pathway in colorectal cancer. Oncol Rep. 2019; 41 (5): 2717-2728.

28. Yang Z, Zhang C, Qi W, Cui Y, Xuan Y. GLI1 promotes cancer stemness through intracellular signaling pathway $\mathrm{PI} 3 \mathrm{~K} / \mathrm{Akt} / \mathrm{NFKB}$ in colorectal adenocarcinoma. Exp Cell Res. 2018; 373: 145-154.

29. Lin WF, Lin XL, Fu SW, Yang L, Tang CT, Gao YJ, Chen HY, Ge ZZ. Pseudopod-associated protein KIF20B promotes Gli1-induced epithelial-mesenchymal transition modulated by pseudopodial actin dynamic in human colorectal cancer. Mol Carcinog. 2018; 57(7): 911-925.

30. Wang C, Cheng L, Song S, Wu S, Sun G. Gli1 interacts with YAP1 to promote tumorigenesis in esophageal squamous cell carcinoma. J Cell Physiol. 2020; 235(11): 8224-8235.

31. Han $\mathrm{T}$, Cheng $\mathrm{Z}, \mathrm{Xu} \mathrm{M}$, Wang $\mathrm{X}, \mathrm{Wu} \mathrm{J}$, Fang $\mathrm{X}$. Yes-Associated Protein Contributes to Cell Proliferation and Migration of Gastric Cancer via Activation of Gli1. Onco Targets Ther. 2020; 13: 10867-10876. 